\section{Win with ProTaper Universal!}

DENTSPLY are giving you the chance to win a trip to Universal Studios, Florida with ProTaper Universal root canal treatment systems. The lucky winner will have the chance to walk through full-scale movie sets as well as enjoying the exciting rides and shows.

Built upon the enormous success of the existing ProTaper range, the Universal system has been enhanced and is one of the most simple, efficient and safe systems on the market. The range now includes treatment files, re-treatment files and dedicated obturation systems. Due to their unique multi-tapered shape, only three ProTaper treatment files are needed to efficiently and effectively shape the majority of canals for optimal cleaning and true three-dimensional filling.

For your chance to win, simply contact Kathryn Norris at DENTSPLY for your entry form on 01932837313 or by email on kathryn.norris@dentsply.co.uk. Reader response number 51

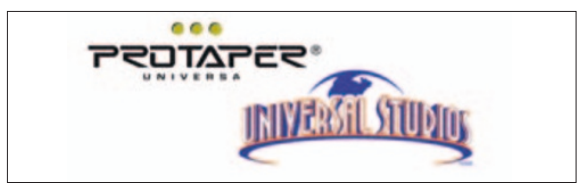

\section{Riva Light Cure}

Riva Light Cure from SDI Ltd has the advantages of a true glass ionomer with the aesthetic, life-like look of a composite. A resin reinforced glass ionomer restorative, it has all the strength, chemical adhesion, fluoride release and biocompatibility that you require. In addition, Riva Light Cure has brilliant aesthetics to give restorations a life-like quality.

A wonderful restorative for deciduous and geriatric teeth, Riva Light Cure takes seconds to place and no etching, bonding or varnishing is needed. It is also excellent used as a liner or a base, or for class II, $\mathrm{V}$ and small class I and II restorations.

For more information, please contact SDI Ltd on 080002255734 or visit www.sdi.com.au.

Reader response number 52

\section{TRADE NEWS WHAT'S NEW}

\section{Near perfect results}

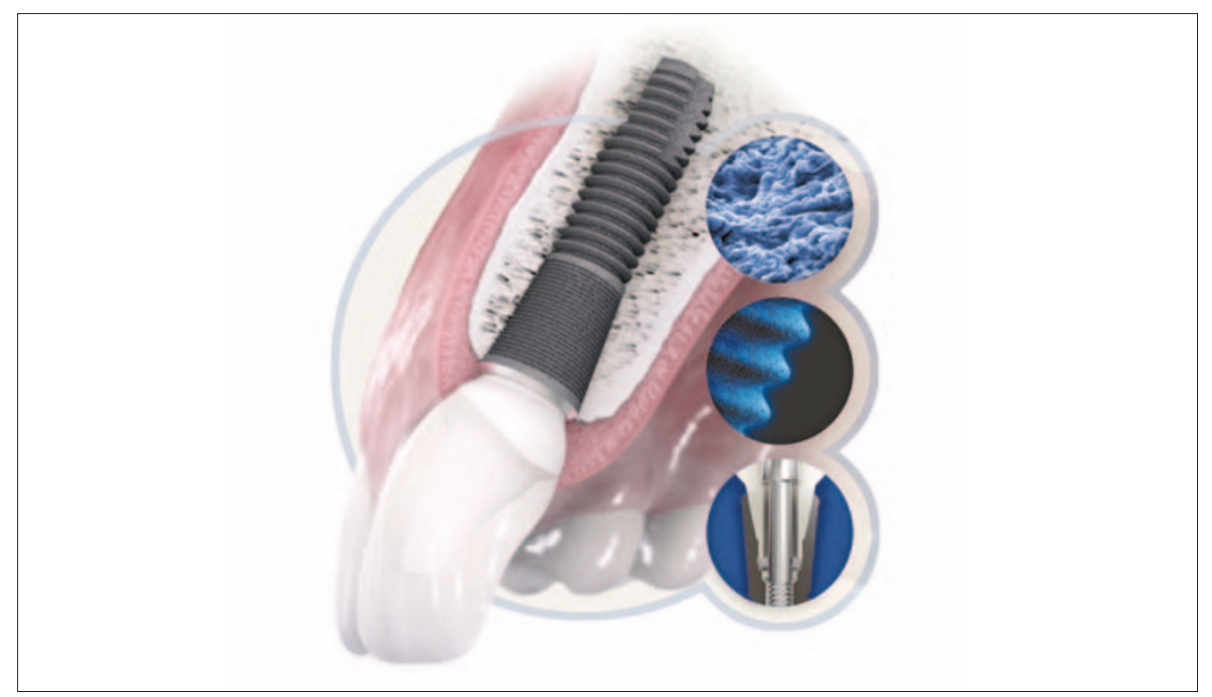

Astra Tech's implant system has been developed after 15 years of dedicated research and places the company firmly at the vanguard of clinical dental innovation. Now Astra Tech has announced an incredible success rate of $98.6 \%$ for their system, revealed in an international study across 15 countries.

The result was announced at the Astra Tech World Congress in New York in April, and is further proof that Astra Tech delivers on its promises. Dental professionals worldwide have ready access to an implant system which widens the parameters of possibility and UK clinicians using the Astra Tech system will now work with reinforced confidence. Astra Tech continues to pursue a policy of ongoing development, high quality customer service and genuinely cost effective implant products. For more information on the Astra Tech Implant System please call 01453791763.

Reader response number 50

\section{'Custom Care' from Sonicare}

Sonicare's new Elite e9000 range has been specially designed around the individual needs of each patient.

Two contoured brush heads are provided so that each patient can choose the head that best suits their needs: a standard brush head and a new smaller brush head designed to suit children, those with smaller mouths and young people undergoing orthodontic treatment. The technologically advanced handle has dual speed options - 'max' and 'gentle' - to allow the brusher to customise their brushing experience. A Smartimer ${ }^{\circledR}$ feature encourages two minutes of accurate timed cleaning, helping patients keep to their dentists' recommended brushing time, and a programmable Quadpacer function is also included to encourage equal importance to be placed on brushing each quadrant of the mouth effectively.

The new e9000 is available for dental professionals and can be sold directly from your practice to the patients. For more information please contact Tracy Posner on 02085668811 or email tracyposner@btconnect.com.

Reader response number 53

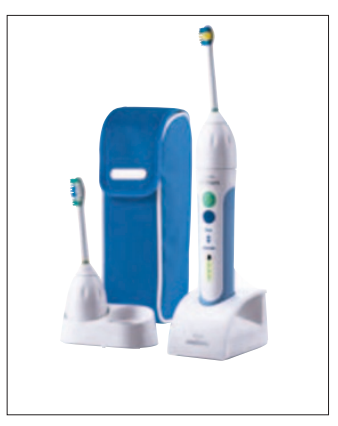



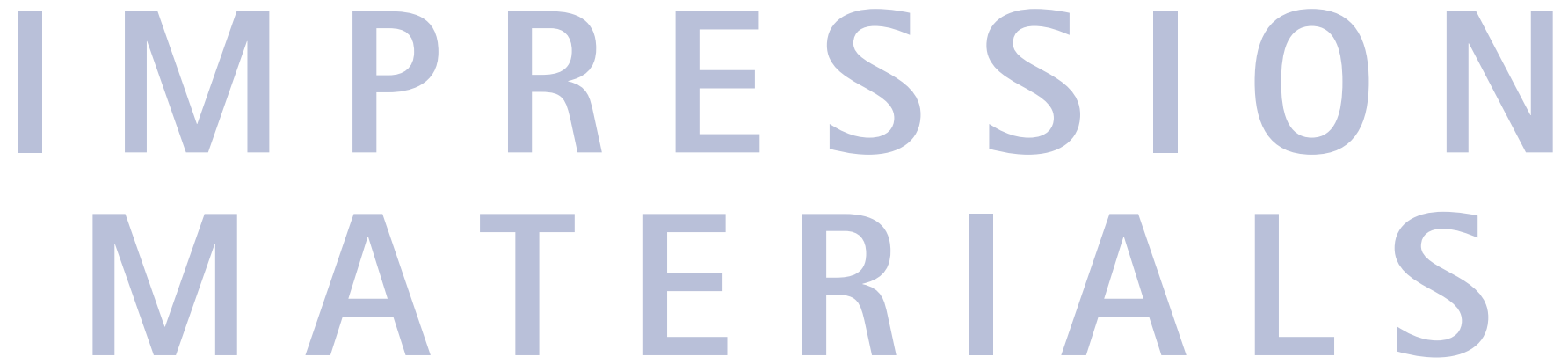

\section{Impression materials to impress!}

Dental Sky supplies an impressive range of leading brand impression materials as well as its own RetS Turboflex. REtS Turboflex is the perfect addition cured silicone that provides exceptional detail reproduction. The complete range allows you to use your existing technique, whether it is the wash technique or double mixing.

The light bodied materials within the range are easily injected and do not slump, even when placed in the upper arches. With extremely high levels of hydrocompatibility, Turboflex will always provide precise impressions, even when in contact with gingival fluid and blood. In addition, Turboflex has very good dimensional stability and high elastic memory, making removal of the impression simple. The material is so stable that several models can be made from a single impression without any risk of deformation.

Whether you choose your existing brand of impression material or would like to try Dental Sky's cost effective Turboflex, call Dental Sky on 08002944700.

Reader response number 55

\section{DENTALSKY}

\section{Revolutionising alginate impressions}

Alfa Tray is the latest addition to the Triple Tray ${ }^{\circledR}$ family from Premier ${ }^{\circledR}$. Easy to use and producing excellent results, the Alfa Tray is indicated for full arch alginate impressions for the fabrication of custom trays, study models, night guards, sports guards, whitening trays and as a matrix for professional restorations.

The unique palate-free, patent pending design engages the patient's tongue to restrict the overflow of material and eliminate gagging. The patient's mouth is also closed in a more natural, comfortable position. Because the Alfa tray simultaneously captures upper and lower impressions plus the bite registration, significant time is saved and about half the amount of alginate used in existing techniques is required. In addition, the Alfa Tray can be operated hands free and is disposable, so avoiding cross contamination. Only four steps are required between measuring and mixing the alginate and pouring the models.

For further information please contact Myerson on 02088639044.

\section{Reader response number 56}

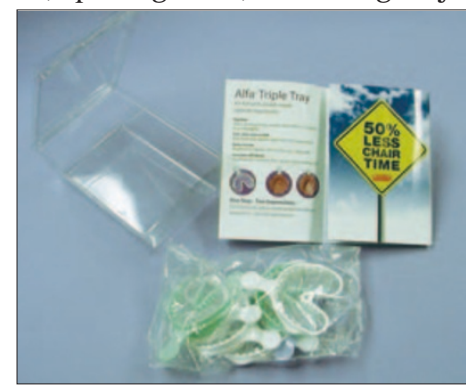




\section{AlgiNot, not alginate!}

Until recently, alginate has been the only viable option for certain dental impressions. While fast setting times, mild flavours and low cost have long been alginate's attractive qualities, the downsides include poor dimensional stability, hazardous dust, messy and repetitive hand mixing and a lot of wasted time. Now there's a better way - AlgiNot ${ }^{\mathrm{TM}}$ from Kerr.

AlgiNot is an extremely cost effective, reliable alternative to alginate that delivers exceptional accuracy and performance. Presented in self-mixing cartridges, it is simple to use and saves the dental assistant a tremendous amount of time and energy. Quite simply, AlgiNot is revolutionary! For further information please contact Kerr UK on 01733892292.

Reader response number 57

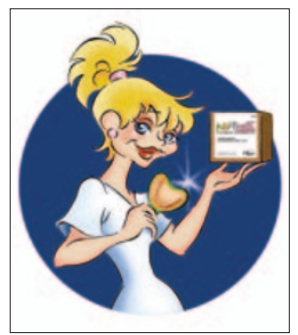

\section{Faster setting}

GC has produced a range of impression materials that set in super fast time. The Exafast NDS family of addition cured silicones provide high quality, accurate and consistent impressions every time. Exafast NDS allows you to produce very smooth and highly precise impressions in only two minutes, thus improving productivity and maximising the comfort of your patients.

GC Exafast putty is an extremely fast setting putty material, ideal for use in the single step technique. In combination with other Exafast NDS materials it offers the perfect combination of speed and precision. Exafast wash material is available in three viscosities to

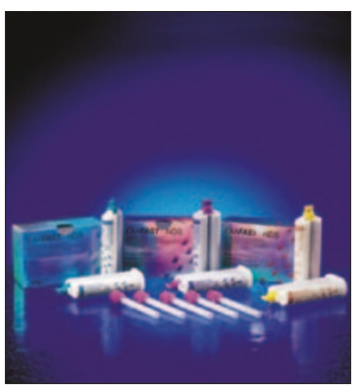
suit your preferred technique and all are supplied in cartridges for speed and ease of application. As the material sets so quickly there is less risk of distortion due to movement during setting.

With a high degree of elasticity, excellent tear resistance and good dimensional stability, Exafast NDS ensures that your impressions are perfect every time.

For more information contact GC UK Ltd on 01908218999 or email gcuk@btinternet.com.

Reader response number 58

\section{TempSpan for perfect temporaries}

TempSpan Clear Matrix is a clear, medium viscosity vinyl polysiloxane that reproduces the finest detail, resulting in extremely accurate provisional restorations. Automixed from $50 \mathrm{ml}$ cartridges and with just two minutes oral setting time, it is a significantly faster, more reliable and easier alternative to vacuum formed stents, alginate and handmixed putties.

Fast and accurate, TempSpan Clear Matrix also enables dual cure temporary crown and bridge materials to be light cured both intra and extra-orally. This drastically shortens the procedure, minimising chair time and patient discomfort. For further information on TempSpan Clear Matrix, contact Trycare Ltd on 01274881044 or email info@trycare.co.uk. Reader response number 59

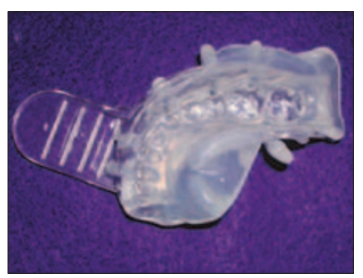

\section{Dedicated impression disinfection}

Perform ${ }^{\circledR}$ ID from Schülke \& Mayr is a high level disinfection product for impressions, specially formulated to respect the dimensional stability of materials such as alginates, silicones and polyethers. Quick and easy to use, the impressions just need to be soaked in the solution for 10 minutes.

Perform ${ }^{\circledR}$ ID is active against bacteria (including TB), fungi and viruses including HIV, HBV and Polio in 10 minutes at 2\% dilution. The in-use solution remains active for 24 hours.

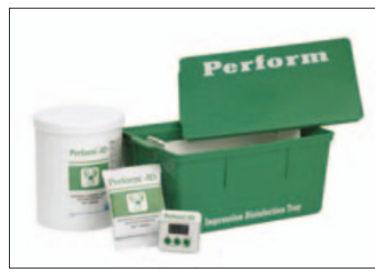

CE marked in line with European directives, Perform ${ }^{\circledR}$ ID fully complies with the BDA A12 guidelines on impression disinfection. The product is available in both $900 \mathrm{~g}$ tubs and 40 g sachets.

For more information please contact Schülke \&t Mayr on 01142543500 or visit www.schulke-mayr.com.

Reader response number 60 